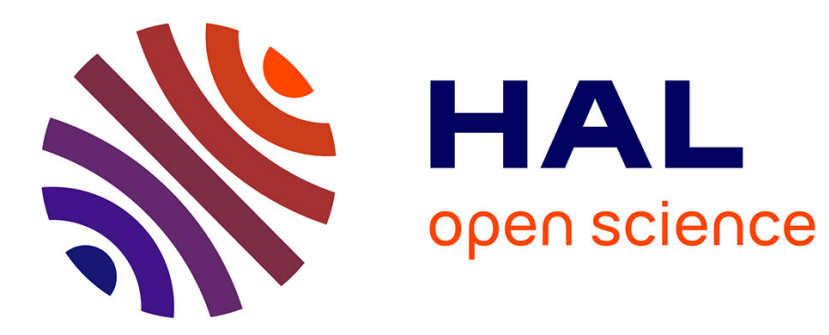

\title{
Real Space Observation of Electronic Coupling between Self-Assembled Quantum Dots
}

Guillemin Rodary, Lorenzo Bernardi, Christophe David, Bruno Fain, Aristide Lemaitre, Jean-Christophe G Girard

\section{- To cite this version:}

Guillemin Rodary, Lorenzo Bernardi, Christophe David, Bruno Fain, Aristide Lemaitre, et al.. Real Space Observation of Electronic Coupling between Self-Assembled Quantum Dots. Nano Letters, 2019, 19 (6), pp.3699-3706. 10.1021/acs.nanolett.9b00772 . hal-02337790

\section{HAL Id: hal-02337790 \\ https://hal.science/hal-02337790}

Submitted on 31 Oct 2019

HAL is a multi-disciplinary open access archive for the deposit and dissemination of scientific research documents, whether they are published or not. The documents may come from teaching and research institutions in France or abroad, or from public or private research centers.
L'archive ouverte pluridisciplinaire HAL, est destinée au dépôt et à la diffusion de documents scientifiques de niveau recherche, publiés ou non, émanant des établissements d'enseignement et de recherche français ou étrangers, des laboratoires publics ou privés. 


\title{
Real space observation of electronic coupling
}

\section{between self-assembled quantum dots}

\author{
Guillemin Rodary*, Lorenzo Bernardi†, Christophe David, Bruno Fain ††, Aristide Lemaître, \\ Jean-Christophe Girard
}

Centre de Nanosciences et de Nanotechnologies (C2N), CNRS, Université Paris-Sud, 10 Boulevard Thomas Gobert, 91120 Palaiseau, France.

\begin{abstract}
The control of quantum coupling between nano-objects is essential to quantum technologies. Confined nanostructures, such as cavities, resonators or quantum dots, are designed to enhance interactions between electrons, photons or phonons, giving rise to new properties on which devices are developed. The nature and strength of these interactions are often measured indirectly on an assembly of dissimilar objects. Here, we adopt an innovative point of view by directly mapping the coupling of single nanostructures using Scanning Tunneling Microscopy and Spectroscopy (STM and STS). We take advantage of the unique capabilities of STM/STS to map simultaneously the nano-object's morphology and electronic density in order to observe in real space the electronic coupling of pairs of $\operatorname{In}(\mathrm{Ga}) \mathrm{As} / \mathrm{GaAs}$ self-assembled Quantum Dots (QDs) forming Quantum Dots Molecules (QDMs). Differential conductance maps dI/dV(E, x, y) demonstrate the presence of an effective electronic coupling leading to bonding and antibonding states, even for dissymmetric QDMs. The experimental results are supported by numerical
\end{abstract}


simulations. Actual geometry of the QDMs is taken into account to determine the strength of the coupling, showing the crucial role of quantum dot size and pair separation for devices growth optimization.

KEYWORDS: quantum dots molecules, quantum information, scanning tunneling microscopy and spectroscopy, electronic properties

\section{Introduction}

Semiconductor Quantum Dots (QDs) have been extensively studied because of their attractive atom-like electronic properties. In the context of quantum computing and quantum information, chains of coupled QDs, i.e. quantum dots molecules (QDMs), could realize the key building block of a quantum device, the qubit. ${ }^{1,2}$ One main requirement for such an application is that the QDs be electronically coupled. Self-assembled $\operatorname{In}(\mathrm{Ga}) \mathrm{As} / \mathrm{GaAs}$ QDs grown by molecular beam epitaxy have appeared as interesting objects to implement these blocks. ${ }^{1,3-6}$ Indeed, stacks of vertically coupled QDs can be grown thanks to the strain field which drives the growth of the upper dot on the top of the lower QDs. ${ }^{7}$ The electronic coupling has already been demonstrated by optical means and by several teams ${ }^{4,8-10}$ in case of double dots. However, inherently to selfassembled growth mode, the QDs forming the molecule are usually asymmetric, with nonequivalent sizes, which strongly affect the coupling strength. ${ }^{11}$ To get a better knowledge of this coupling, one needs to relate the structural characteristics to the QDM electronic structure. Scanning Tunneling Microscopy and Spectroscopy (STM and STS) have greatly contributed to the characterization of single QD morphology and electronic structure, ${ }^{12-17}$ as well as coupled quantum well and QDM structures. ${ }^{18-20}$ In order to study the electronic coupling between QD pairs, 
controlling simultaneously their morphology at the atomic level, Fölsch and al. have proposed an attractive strategy by measuring QDMs formed by chains of atoms created by STM atomic manipulation. ${ }^{21}$ Here, we present a complementary approach where accurate structure and electronic coupling between QDs are studied concurrently by cross-sectional STM (X-STM) in a realistic system, i.e. pairs of non-identical self-assembled epitaxial QDs embedded in a semiconductor heterostructure, as they are used in QDs based device. Different coupling regimes are identified depending on the QD pair specific morphology (size, shape and interdots separation).

\section{Sample and experimental system}

QD pairs were grown at $500^{\circ} \mathrm{C}$ by molecular beam epitaxy on a (001) oriented GaAs substrate. The sample consists of a set of double $\operatorname{In}(\mathrm{Ga})$ As QD layers separated by GaAs spacers. The whole structure is highly p-type doped $\left([\mathrm{Be}]=2.10^{-19} \mathrm{~cm}^{-3}\right)$ to ensure electrical conductivity. The interdot separation and the nominal thickness of the InAs QD layer were chosen to investigate different QDM structures by X-STM: the nominal GaAs thickness between two InAs layers varies from 4 monolayer (ML) to $9 \mathrm{ML}$; the nominal thickness of the first InAs layer is $2 \mathrm{ML}$ while the second layer varies from 1.4ML to 1.7ML. Samples are cleaved in situ under ultra-high vacuum $\left(<8.10^{-11} \mathrm{mbar}\right)$ and transferred rapidly into the STM chamber to be measured at $4.2 \mathrm{~K}$. The cleaving procedure gives access by X-STM to the sample cross-section, i.e. the GaAs(110) surface

perpendicular to the growth direction. ${ }^{15}$ See Methods section for additional information on experimental details.

Figure 1 shows a STM constant current image of a large area including four QD pair layers. The pairs appear as red areas embedded in yellow lines corresponding to the QD wetting layers. A 
zoom on a QD pair surrounding by the GaAs matrix and the $\operatorname{In}(\mathrm{Ga})$ As wetting layer is presented in the inset of Fig. 1 where atomic pattern is resolved.

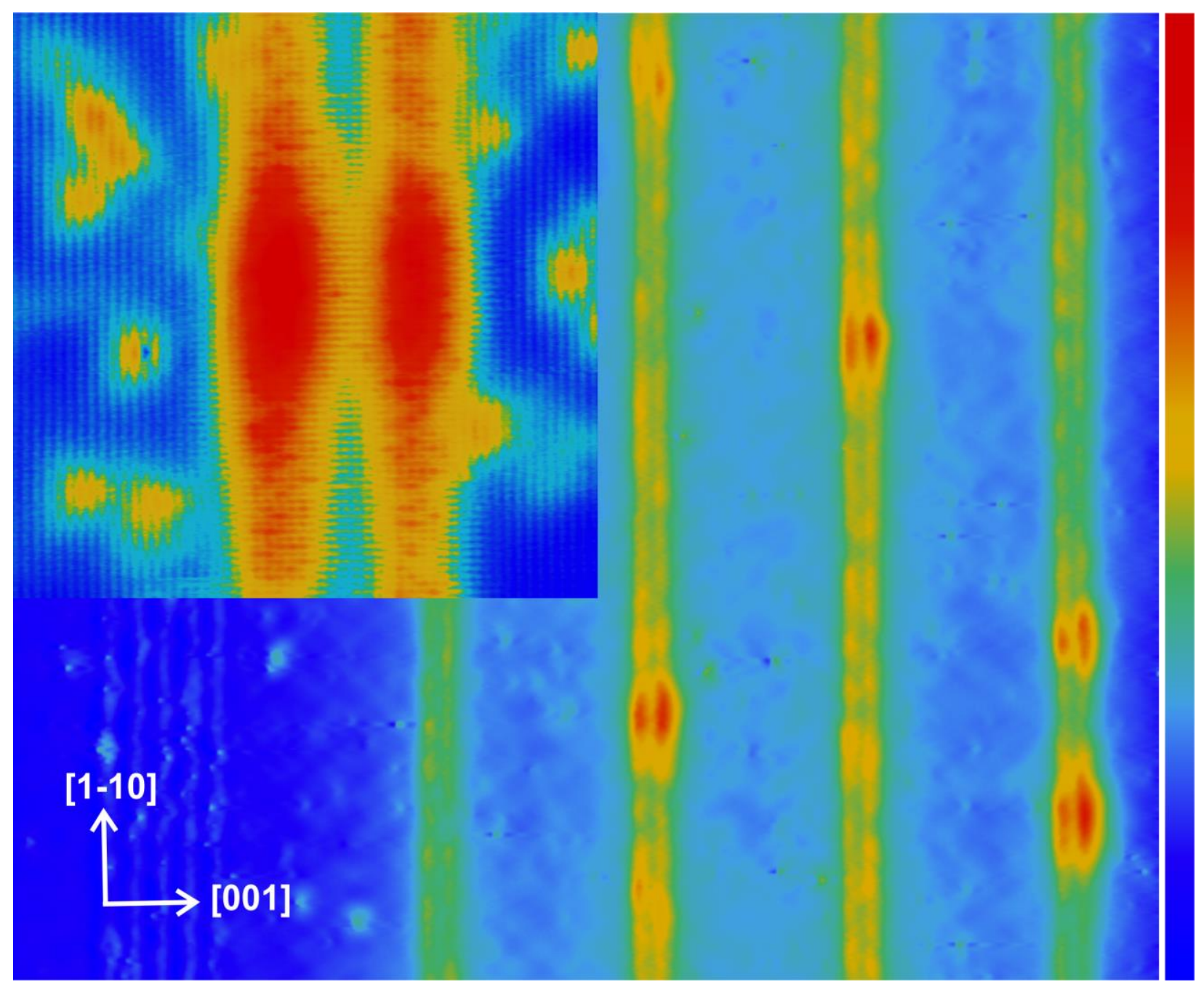

Figure 1. STM image of quantum dots pairs. STM constant current large scale image of a $225 \mathrm{~nm} \times 190 \mathrm{~nm}$ area where four double QD layer are observed. Blue background corresponds to GaAs matrix, yellow lines correspond to InGaAs QD wetting layers and red protusions to double $\operatorname{In}(\mathrm{Ga})$ As QDs. Bright vertical lines on the left hand are markers allowing a quick reconnaissance of the sample area. (Image parameter: $1.55 \mathrm{~V}, 300 \mathrm{pA}$, z-range of the whole color bar: 667 pm). Inset: zoom on a QD pair with atomic resolution. Yellow triangles around the QD are Be dopants. The red atomic scale dots observed in the $\operatorname{In}(\mathrm{Ga})$ As layer are ascribed to In atoms present in the QD and in the wetting layer (30 nm x30 nm, $1.65 \mathrm{~V}, 300 \mathrm{pA}$, z-range: $565 \mathrm{pm}$ ). 


\section{Electronic coupling between quantum dots}

We explored the electronic properties of QD pairs of several sizes and interdot distances (from center to center) by measuring the density of state by STS, i.e. the differential conductance $\mathrm{d} / \mathrm{dV}(\mathrm{V})$ as a function of the voltage. ${ }^{22}$ Figure 2 presents STM images ( $\mathrm{a}, \mathrm{b}$ and $\mathrm{c}$ ) and STS spectra (d, e and f) of three significant examples of QDs separated by 15 ML (a and d), 12 ML ( $b$ and e) and $7 \mathrm{ML}$ (c and f), i.e. $8.48 \mathrm{~nm}, 6.78 \mathrm{~nm}$ and $3.96 \mathrm{~nm}$ respectively. This corresponds to GaAs spacers of 9 ML, 6 ML, and 4 ML. The spectra on Fig. $2 d$ (respectively $2 \mathrm{e}$ and 2f) were acquired at the center of both QDs shown on Fig. 2a (respectively $2 \mathrm{~b}$ and $2 \mathrm{c}$ ). They present sharp peaks, and a shoulder, i.e. an increase of the differential conductance above $1.45 \mathrm{~V}$. The peaks correspond to high density of state localized in the QDs and are ascribed to discrete quantized states, ${ }^{15}$ while the shoulder arises from tunneling into the continuum of states of the conduction band. The Fermi level is located at the zero bias, and is here at the top of the valence band as the structure is heavily p-doped. Contrary to colloidal QDs, no hole states in the valence band have been observed in this study and other previous works on self-assembled epitaxial QDs and quantum wells ${ }^{14-16,20}$. Indeed, the measurement geometry is very different for the two type of QDs. In the case of self-assembled QDs appearing at a semi-conducting surface in X-STM, the Tip Induced Band Bending (TIBB) ${ }^{23}$ effect shifts strongly the band structure, even à $\mathrm{V}=0$, which hindered the hole states towards the negative voltages.

The three QD pairs show noteworthy different behaviors. In the most separated case (Fig. 2a and 2d), a single marked peak is measured on each spectrum. They are ascribed to the ground state of individual, uncoupled QDs. The peak position depends on the QD thickness (the QD on the right of the Fig. 2a is slightly thicker than the one on the left): the thicker QD shows a ground state at 
lower energy as compared to the smaller QD, as expected theoretically for an isolated QD. ${ }^{24} \mathrm{In}$ this case, the two QDs are too far apart from each other to interact. The electronic coupling is weak and therefore the discrete states are independent in both QDs: no electronic signature of the first QD state is measured at the second QD position, and reciprocally. Figure $2 \mathrm{~g}$ gives a schematic view of this case of two uncoupled (or weakly coupled) QDs. The states measured for this QD pair have the same energy than the states of isolated QD taken individually.
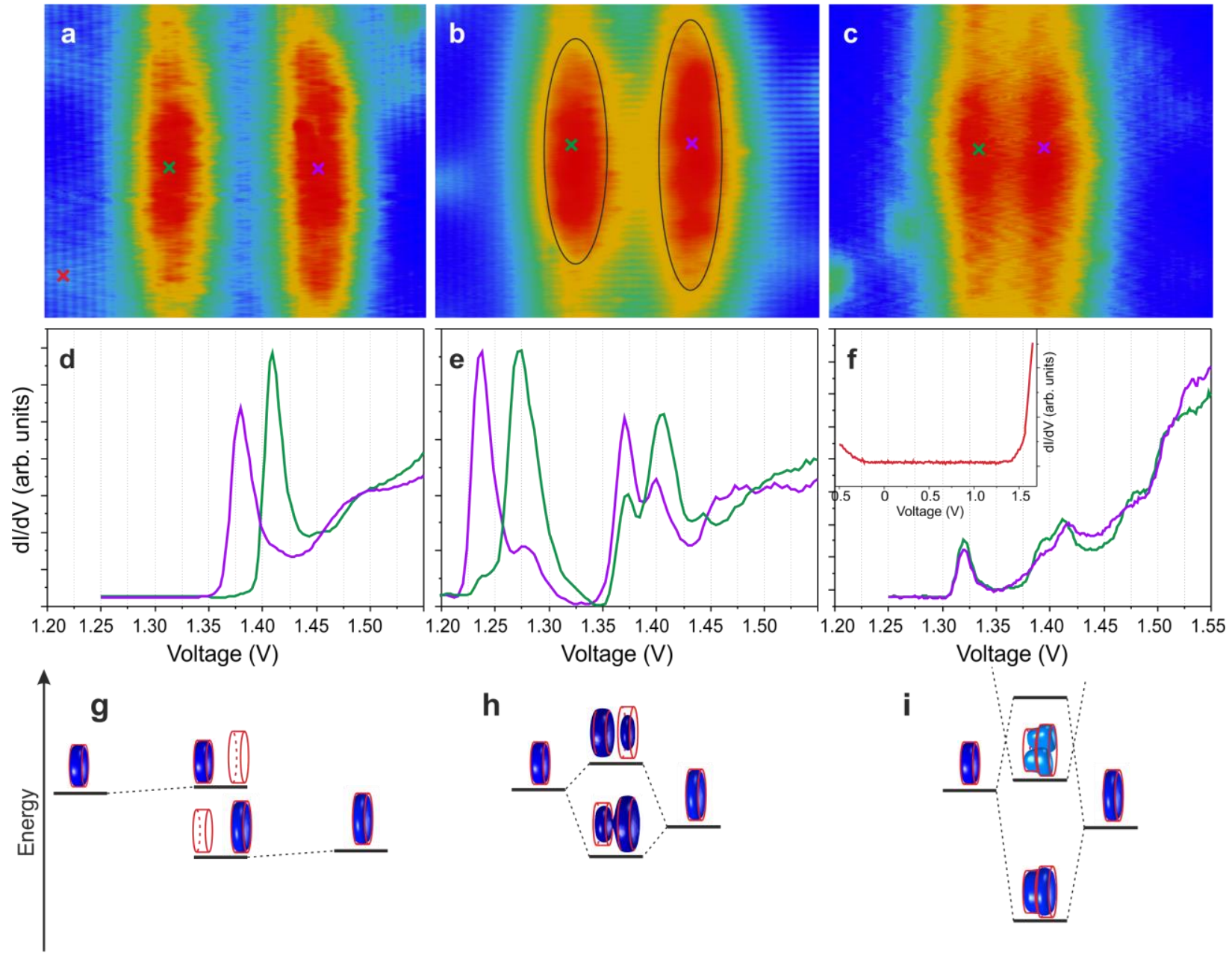

h

\section{Weak coupling}
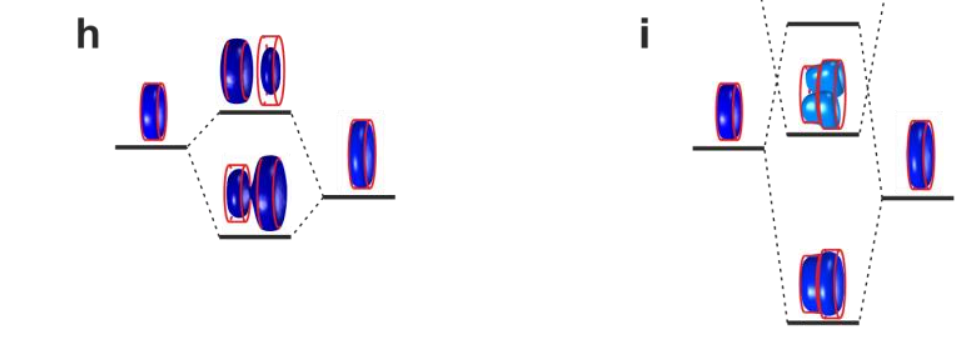

Intermediate coupling

\section{Strong coupling}

Figure 2. Spectroscopy on quantum dot molecules of different coupling. a, b and $\mathbf{c}$ : STM constant current image of pairs of QD separated by 15ML, 12ML and 7ML respectively (22 nm x $18 \mathrm{~nm}, 1.55 \mathrm{~V}, 300 \mathrm{pA})$. Ellipses in b mark the topographic contour of the cleaved QD. d, e and f: STS spectra measured on the center of the QD of Fig. 2a, $\mathbf{b}$ and 6 
c respectively. Colored crosses indicate the position where each spectrum (of the same color) have been recorded. The first case (Fig. 2a and 2d) corresponds to a weak electronic coupling, the second case (Fig. 2b and 2e) to an intermediate coupling, and the third one (Fig. 2c and $2 \mathbf{f}$ ) to a strong coupling. Figures $\mathbf{g}, \mathbf{h}$ and $\mathbf{i}$ represent a schematic view of the coupling mechanism give rise to bonding and antibonding states splitting. Inset of Fig. $2 \mathbf{f}$ shows dI/dV spectra measured on bare GaAs surface.

In the case of closer QDs (Figures $2 \mathrm{~b}$ and $2 \mathrm{e}$ ), two main peaks, separated by approximately $135 \mathrm{mV}$, are observed on each spectrum measured at the center of both QDs ascribed to the ground state and the first excited state. Our instrumental energy accuracy is less than $1 \mathrm{mV}$, and our measurement precision of the conductance peaks is given by the broadening due to the temperature and the lock-in modulation technique (see Methods). In addition, the width of the conductance peaks depends also on physical phenomena such as the interaction of the electrons on these states with other bulk or nearby electronic states. The QDs are here slightly larger than in case $2 \mathrm{a}$ which explain the red shift of the ground state energies. This red shift can also be explained by the coupling itself between the QDs. ${ }^{25,26}$ For each spectrum taken at a QD center, smaller peaks (or a shoulder) appear close to the main peaks. They arise at the same energy as the second QD main peaks on the other spectrum. These double peaks suggest that the electronic states are partly delocalized over the two QDs, a signature of the electronic coupling occurring between these two QDs. As we shall confirm later when probing the wave function amplitude, the first pair of peaks at $1.238 \mathrm{~V}$ and $1.271 \mathrm{~V}$ correspond to the bonding and anti-bonding molecular states. The energy splitting between these molecular states, around $30-40 \mathrm{mV}$, is quite smaller than the energy splitting, 60-100 mV, between the ground and excited states of single QDs. ${ }^{15}$ For two strictly equivalent QDs, the electron density is symmetrical over the two dots, for both the bonding and the antibonding states. However, in realistic cases, QDs are dissimilar, and the electronic density 
is then expected to be different for a given state from one dot to the other, in agreement with our observation: due to size difference between dots (Fig. 2b), the bonding state measured at $1.238 \mathrm{~V}$ (respectively the antibonding state at $1.271 \mathrm{~V}$ ) present a much smaller (respectively larger) peak for the smaller QD as the one for the larger QD (Fig. 2e). Figure 2h gives a schematic interpretation of this effect. Due to the proximity of individual QD states, they hybridize, giving rise to molecular states with bonding state (lower in energy) and antibonding state (higher). The bonding state (respectively antibonding state) arises closer in energy to the state of the larger (respectively smaller) isolated QD state, taking therefore its character ( $\mathrm{S}, \mathrm{P}$ or D type). It means that the electronic density weight is localized preferentially on one QD. Following previous experimental and theoretical works, ${ }^{12,}$ 24, 27, 28 we use " $S$ " and " $P$ " notation to describe our waves functions observation. Several notations exist and we choose to use the one used for single particle state in spherical systems since it allows to describe simply for the readers the wave function shape: 0 nodal plane corresponds to S-like state and 1 nodal plane corresponds to P-like state.

In the last case of very close QDs, the GaAs spacer layer is no longer observed and the two QDs merge (Fig. 2c). The first states observed by STS at the two QDs center positions have the same energy and intensity (Fig. 2f). In this situation, the coupling is strong and the states are delocalized indistinctly over the whole pair. The two QDs behave as a complex system and the ground state energy position at $1.32 \mathrm{~V}$ results of the merging of two QDs smaller than the one of Fig. 2a and $2 \mathrm{~b}$. The peaks observed between $1.38 \mathrm{~V}$ and $1.5 \mathrm{~V}$ might be a family of unresolved states due to experimental energy broadening (see Methods). Due to the strong coupling, the energy difference between bonding and antibonding states is large and states of different characters may appear below the anti-bonding state as schematized in Fig. 2i. 


\section{Waves functions mapping}

One major interest of mapping the spatial distribution of the molecular bonding and antibonding states is the identification or not of nodes into the delocalized wave functions of the QDM. $^{21,29}$ We should note that unlike optical spectroscopy, STM gives rise to injection of electrons in the empty states of the conduction band without exciton creation. The resulting wave function measured by STS is therefore the one of an electron rather than the one of two correlated hole and electron. However, for two closely separated QDs, we cannot exclude Coulomb interaction effects influencing the wave function, which is beyond the scope of this work. Figure 3a represents a mapping of the differential conductance measured along a line crossing the two coupled QDs at their center (as visualized in Fig. 3d) as a function of the energy and the position along that line. This figure gives a direct visualization of the distribution of the electron density for a given state as a function of the energy and position. $\mathrm{dI} / \mathrm{dV}(\mathrm{V})$ spectra of Fig. 3b, taken along the two vertical lines in Fig. 3a, show that the QDM behaves in a similar way as the one presented on Fig. $2 b$ and 2e, i.e. with intermediate coupling. The electron density profile is shown in Fig. 3c for the four first levels. Both the ground state (solid lines) and the first exited state (dashed lines) are split into two levels: the bonding states (yellow curves), and the antibonding states (red). The shape of the lobes confirms what has been described in the spectra of Fig. 2e: the states are delocalized over the two QDs: the electron density is higher in the larger QD for the bonding states and in the smaller QD for the antibonding states. The density vanishes in between the two QDs for the antibonding state, revealing the node at the QDM center at an antibonding wave function. On the opposite, the density remains non-zero for the lower state over the full QDM width, as expecting for the bonding wave function. 




Figure 3. Bonding and antibonding state visible from spectroscopy mapping. a: differential conductance $\mathrm{d} / \mathrm{dV}$ dependence with distance (X-axis) and voltage (Y-axis) measured along the black line on the QDM shown in $\mathbf{d}$ (22 $\mathrm{nm}$ $\mathrm{x} 18 \mathrm{~nm}) . \mathbf{b}$ and $\mathbf{c}$ represent cross-sections of Fig. $3 \mathbf{a}$ along the voltage axis and along the distance axis respectively. Green and purple spectra in $\mathbf{b}$ are profiles measured at the center of the two QD as indicated by vertical lines in $\mathbf{a}$ of the same colors. Yellow and red curves (solid and dash line) in $\mathbf{c}$ are profiles measured along the horizontal lines in $\mathbf{a}$ of the same color and line type (dash lines are shifted upward in $\mathbf{c}$ for clarity). 
We now investigate the in-plane spatial extension of the electron density of the QDM states. We focused on the QDM presented in Fig. 2b and present the results in Fig. 4. The top of the Fig. 4 shows spectra measured on different positions on the two QD: at the center (first lobe, same spectrum as Fig. 2e) and off-center (second and third lobes). We have performed differential conductance grid, i.e. spatial mappings (see Methods for details) on this QDM, which allowed electron density mapping as function of position for a whole range of energy. ${ }^{30}$ Figure 4 (a to e) presents differential conductance maps extracted from this grid at the energy of the QDM discrete states corresponding to peaks position of the spectra of Fig. 4. The state electron density forms lobes localized mainly on one or the other QDs. The first state (Fig. 4a) presents a lobe localized on the larger QD center (at 1.238 V), while the second one (Fig. 4b) is at the smaller QD center (at $1.271 \mathrm{~V}$ ). A weaker lobe is also expected on the opposite QD, as evidenced by the shoulders on the QD center spectra (at $1.238 \mathrm{~V}$ on the green curve and at $1.271 \mathrm{~V}$ on the purple curve): it could not be captured within our signal to noise ratio in Fig. 4a and is visible in Fig. 4b. These first two states are ascribed to the $S$-type ground states, with the bonding state at lower energy and the antibonding state $33 \mathrm{mV}$ above. The next states of type $P$ show two lobes along the vertical direction inside one of the QDs (Fig.4c, right QD, and 4d, left QD) and present a similar behavior but with a larger energy separation: $48 \mathrm{mV}$ between the first bonding state localized mainly on the right QD and the antibonding state mainly on the left QD. The visualization of the electron density of the individual upper states becomes more difficult as the states separation and states broadening energies get close to our experimental grid resolution $(3.6 \mathrm{mV})$. The mapping at $1.374 \mathrm{~V}$ (Fig. 4d) contains contributions from the states measured at $1.370 \mathrm{~V}$ and $1.374 \mathrm{~V}$ from the $\mathrm{dI} / \mathrm{dV}(\mathrm{V})$ spectra, while the mapping at $1.396 \mathrm{~V}$ exhibits contributions from the states centered at $1.396 \mathrm{~V}$ and 
1.407 V. We can however observe that the next states present one single lobe (Fig. 4d right and Fig. 4e left), while the last measured state shows three lobes (Fig. 4e right).
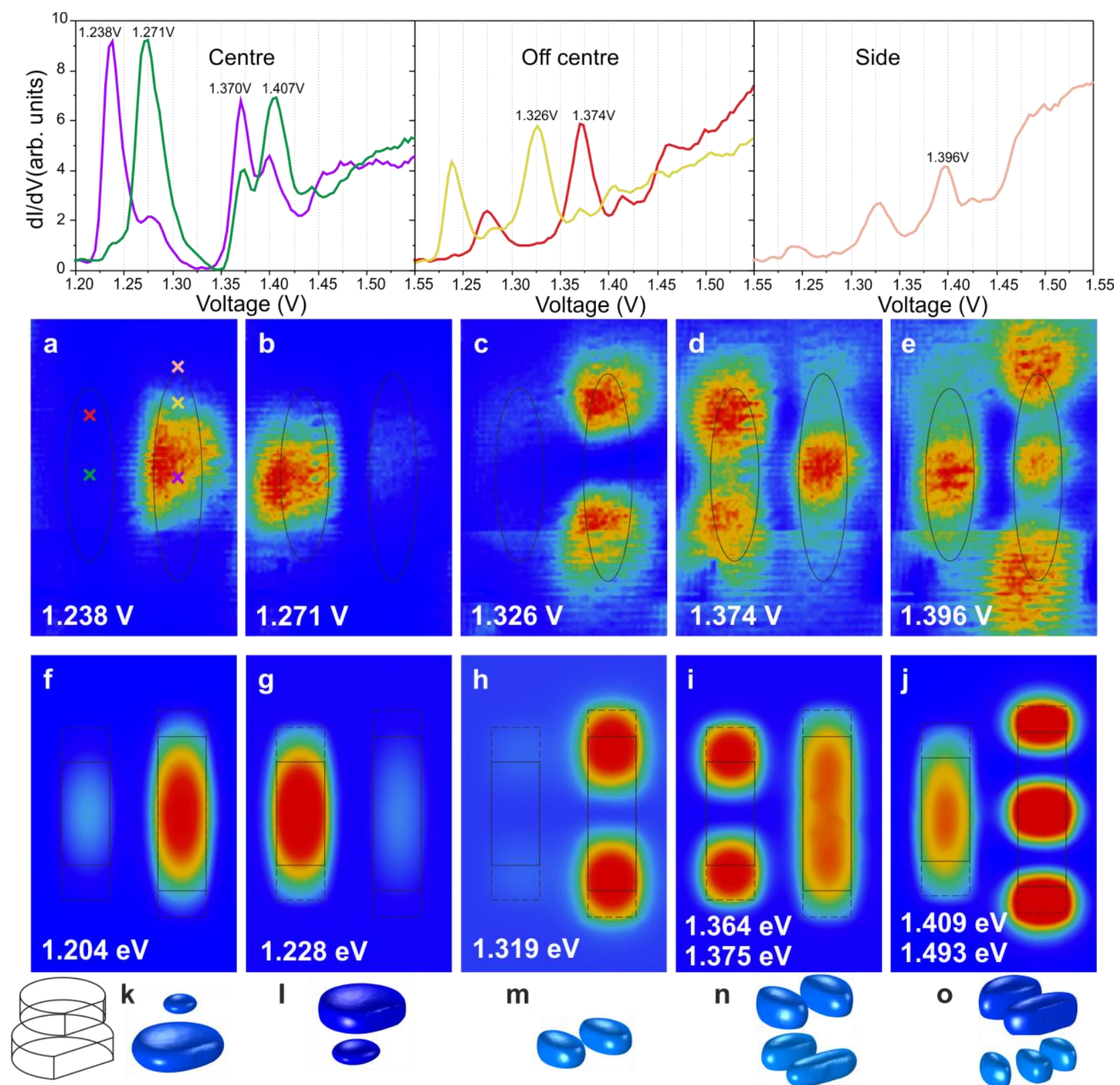

m
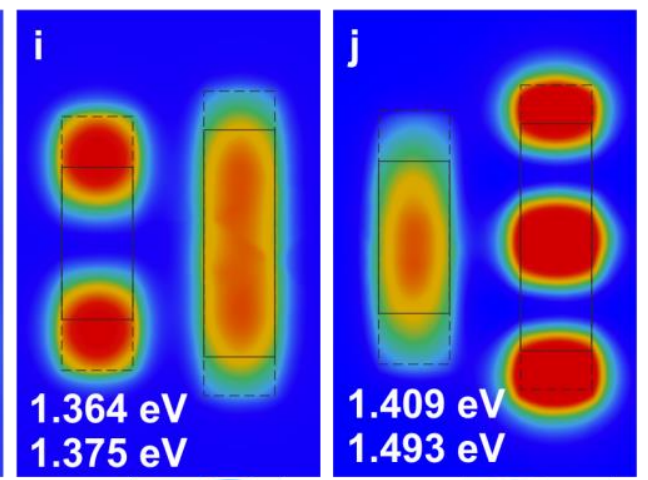

n

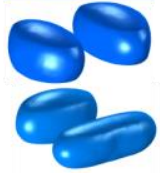

o

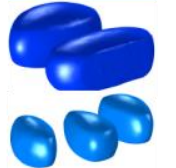

Figure 4. Differential conductance map and simulation. Top row: differential conduction spectra measured at different positions in the QDM. Colors crosses in a indicate measured spectra position. a to e: experimental differential conduction map at spectra peak energy $(15 \mathrm{~nm} \times 23 \mathrm{~nm})$. $\mathbf{f}$ to $\mathbf{j}$ : simulation of the cross-section of the wave function 12 
of the QDM based on the real QDM geometry of the a to $\mathbf{e}$ QD. $\mathbf{i}$ and $\mathbf{j}$ present two states of close energy in the same image for simplicity. $\mathbf{k}$ to $\mathbf{o}$ : 3D simulation of the electronic isodensity of state at the same energy as $\mathbf{f}$ to $\mathbf{j}$.

Let us note that each QD measured in this work presented similar states as those measured in Fig. 4, with one, two or three lobes. Nevertheless, the sequence of state differs from one QDM to another, due to differences in size and QD coupling strength. To disentangled these contributions, we developed both a simple analytical model which allow to understand the global trend of the states position, as well as a numeric simulation which reproduces the main experimental features.

\section{Modelling}

Figure 5a presents the variation with barrier thickness of the two first energy separation (bonding and antibonding $S$-type) obtained experimentally for several QD pairs. Since the respective size of the two QDs are not equivalent for each pair, the data present some dispersion. However, a trend is observed. The splitting between bonding and antibonding levels follows an exponential decrease as a function of the distance between QDs. This trend can be easily reproduced by the textbook model of a one-dimensional double quantum well with infinite walls. If $S_{A}-S_{B}$ is the energy difference between the bonding and antibonding levels, one finds analytically:

$$
S_{A}-S_{B}=\frac{\hbar e^{-K \Delta}}{m K a^{3}}
$$

where $\hbar$ is the Plank constant, $m$ is the effective electron mass in the material, $\Delta$ is the thickness of the barrier separating the two quantum wells and $a$ is the thickness of each well. $K$ is the wave vector in the GaAs barrier, i.e. $K=\sqrt{2 m V_{0}} / \hbar$, $V_{0}$ being the barrier potential. We assume that $K \Delta>>1$ and $K a>>1$. As shown in Fig. 5a, this simple analytical model accounts very well for the 
experimental data when $\Delta$ and $a$ values are taken from STM measurements. Although the double quantum well model cannot provide a detailed simulation of a real system, it gives a good indication of the weight of each parameter that plays a role in QDM coupling, especially the geometrical related parameters.

In order to characterize and identify each state observed, a theoretical approach taking into account the real size and distance between the two QDs should be used. In order to simulate the electronic wave function in the QD, the Schrödinger equation was solved, by finite elements, with the real QDM geometry extracted from STM images (i.e. lateral size, height and interdot distance). The QDs are modelled by cylinders with homogenous electronic properties (see Methods for details). Strain, which reduced the confinement potential, was taken into account using models found in the literature. ${ }^{27,28,31,32}$ Figure 4 present the simulated electronic isodensity of state (Fig. 4k to 4o) and wave function cross-section taken at the QD/vacuum interface (Fig.4f to $4 \mathrm{j}$ ). The simulations were performed for both truncated (due to cleavage) and fully embedded QDMs. A good agreement between the experimental results and simulation for cleaved QD allows to quantify the effect of the cleavage in comparison to how would behave QDMs fully embedded in a semiconductor heterostructure.

Our simulation shows the extreme sensibility of the state positions and sequences on the geometrical parameters, especially the QD interdot distance and their relative size. The sequence is affected from the third or fourth state, for the range of QD sizes probed in this study. In the example of Fig. 4, the simulation agrees qualitatively well with the states observed by STS. The two first states of Fig. 4 correspond to the bonding and antibonding states $S$-type, and the third one is the bonding state of type $P_{x}$. The next state, experimentally measured at $1.374 \mathrm{~V}$ exhibits a single 14 
lobe. A state is also present in our simulation at a similar energy $1.364 \mathrm{eV}$ : a $P_{y}$-type bonding state where the second lobe is located below the surface, and therefore cannot be probed by X-STS (see Fig. $4 \mathrm{~d}_{3}$, bottom). The next simulated states also agree with the experimental results: the $P_{x}$-type $(1.375 \mathrm{eV})$ and $P_{y}$-type $(1.409 \mathrm{eV})$ antibonding states, followed by a state exhibiting three lobes $(1.493 \mathrm{eV})$. We should note that the $\mathrm{TIBB}^{23}$ may explain some discrepancy observed for the energy values between experiments and simulations.

As we have shown above experimentally, an important parameter controlling the coupling between QDs is the thickness of the GaAs barrier separating the QDs. The strength of this coupling will then govern choices in device fabrication based on self-assembled QDMs. Many theoretical studies have therefore addressed the question of states position as a function of barrier thickness. ${ }^{31,33,34}$ However, due to the high sensitivity of the QDMs electronic properties to their real morphology, the prediction of the coupling for a specific QDM requires taking into account the real geometry of the whole system.

To that end, we extend our simulation of the STM results by introducing variations into barrier thickness. Figure $5 \mathrm{~b}$ presents the energy values for each of the first five predicted states of the QDM of Fig. 2b and Fig. 4 as a function of barrier thickness. This QDM presents and interdot distance of $12 \mathrm{ML}$ and a barrier thickness of $6 \mathrm{ML}$, i.e. $3.39 \mathrm{~nm}$. One observes that the barrier thickness changes indeed the position of the energy levels, up to $15 \%$ from $1 \mathrm{~nm}$ to $10 \mathrm{~nm}$ (approximately $2 \mathrm{ML}$ to $18 \mathrm{ML}$ ). Nevertheless, this effect occurs only for closely separated QDs and almost no variation of the level energies is observed beyond a barrier thickness of $4 \mathrm{~nm}$. No state crossing are seen for barrier thickness larger than $1 \mathrm{~nm}$. Beyond this barrier thickness, the level sequence is not controlled by the distance between QD but rather by the QD size. This effect 
is a consequence of the non-equivalence of the two QD sizes that limits the electronic coupling. However, we have shown above that such asymmetric QD pairs are clearly coupled.

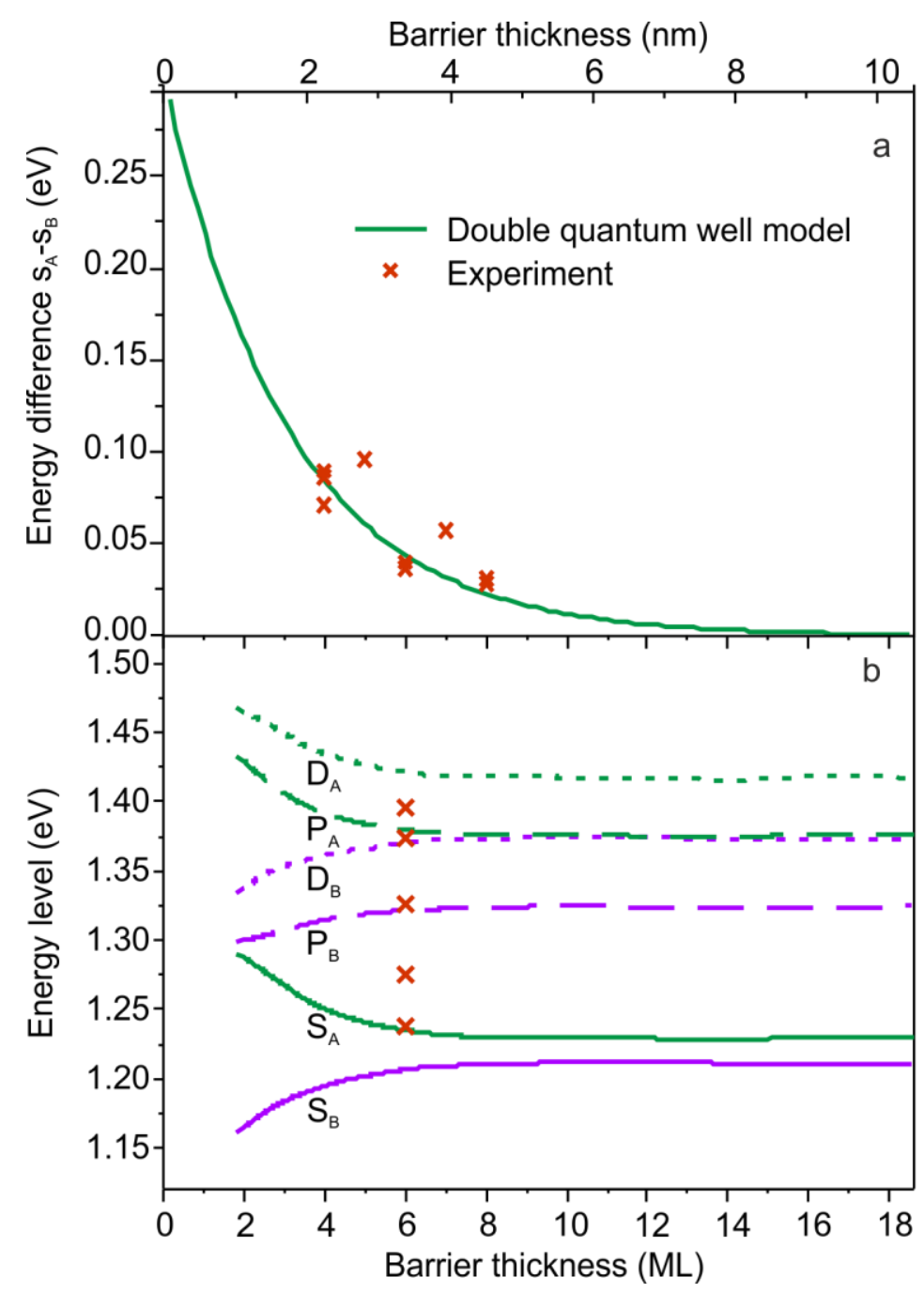

Figure 5. Energy levels as a function of barrier thickness. a: Energy splitting between the first bonding and antibonding states causes by the electronic coupling. Solid line represents the double quantum well analytical model while the crosses represent the experimental data. b: Variation with the barrier thickness of the energy of the first six states ( $S, P$ and $D$ type, B for bonding, and A for Antibonding), calculated for the geometrical parameters extracted from measurement on QD of Fig. 2b. Experimental data are indicated by crosses. 
In conclusion, our study shows the importance of taking into account the real shape, size and interdot distance to determine the electronic coupling strength in QDMs, due to the high sensitivity of the QDMs electronic properties to their real morphology. Although the QDM dissymmetry, inherent to the self-assembly growth mechanism, has been identified as the main difficulty for the fabrication of applied devices with QDMs, we show here that the coupling between QDs can be effective even for non-symmetric QD pairs. Thanks to spectroscopy and wave function mapping by STM/STS, we reveal the electronic coupling in the real space and we observe sequence of bonding and antibonding states. Modelling based on allows us to label the sequences of measured states and to estimate the role and respective contributions of the size and distance between QDs forming the QDM. We believe that our work constitutes an essential step toward the development of qubits with QDMs.

\section{Methods}

X-STM sample preparation. Rectangular pieces of $3 \mathrm{~mm} \times 8 \mathrm{~mm}$ were cut from a thinned p-type $\operatorname{GaAs}((001)$ substrate after MBE growth of the $\operatorname{In}(\mathrm{Ga}) \mathrm{As} / \mathrm{GaAs}$ structure and marked by a notch to guide the in situ cleavage performed under UHV and perpendicular to the long [1-10] axis. Cleaved samples exposing a cross section (110) surface through the QDs structure were transferred within 5 min after cleavage in the precooled low temperature STM head.

STM/STS measurements. Experiments were performed on a LT-STM Omicron system at $4.2 \mathrm{~K}$. Voltage is applied on the sample. Differential conductivity $\mathrm{dI} / \mathrm{dV}$ spectra and grids were directly acquired using a lock-in technique (modulation of $5 \mathrm{mV}$ at $923 \mathrm{~Hz}$ ). The expected energy broadening in this experimental conditions of modulation and temperature is $13 \mathrm{mV} .{ }^{16}$ 
Simulation. Schrödinger equation is solved in the QD using the Comsol software following our previous publication method ${ }^{16}$. The QDs height and lateral size of Fig. 4 is extracted from STM measurement: $3.5 \mathrm{~nm}$ for both QDs and $13 \mathrm{~nm}$ and $15 \mathrm{~nm}$ for the two QDs respectively. Several shapes of the QD has been tried (truncated pyramid, ovoid, cylinder) and no significant difference has been observed on the results. The InGaAs wetting layer surrounding the QD is taken into account. We model the QD by a square potential and in the QD the electronic confinement potential is $0.63 \mathrm{eV}$ and the effective electron mass $0.02 m_{e}$, where $m_{e}$ is the mass of the electron. Reference of energies is taken at the valence band minimum where the Fermi level is positioned.

\section{Corresponding Author}

* Correspondence to: guillemin.rodary@u-psud.fr

Present Addresses

$\dagger$ LLR Ecole Polytechnique, CNRS/IN2P3, 91128 Palaiseau, France

$\dagger \dagger$ CEA, LETI, Minatec Campus, 38054 Grenoble, France.

\section{Author Contributions}

G.R., J.-C.G., C.D and B. F. performed the experiments. GR and J.-C.G. analysed the data. A.L. grew the samples. G.R., L.B. and B.F. performed the calculation. All authors participated in discussion of the results and wrote the manuscript.

\section{Acknowledgements}


This work was supported by a public grant overseen by the French National Research Agency (ANR) as part of the "Investissements d'Avenir" program (Labex NanoSaclay, reference: ANR10-LABX-0035), and by the French National Research Agency (project SSAS, ANR-13-JS100006-01).

\section{References}

(1) Bayer, M.; Hawrylak, P.; Hinzer, K.; Fafard, S.; Korkusinski, M.; Wasilewski, Z. R.; Stern, O. and Forchel, A. Science 2001, 291, 451-453.

(2) Khoshnegar, M.; Huber, T.; Predojevic, A.; Dalacu, D.; Prilmuller, M.; Lapointe, J.; Wu, X. H.; Tamarat, P.; Lounis, B.; Poole, P.; Weihs, G. and Majedi, H. Nat. Commun. 2017, 8, 15716.

(3) Stinaff, E. A.; Scheibner, M.; Bracker, A. S.; Ponomarev, I. V.; Korenev, V. L.; Ware, M. E.; Doty, M. F.; Reinecke, T. L. and Gammon, D. Science 2006, 311, 636-639.

(4) Krenner, H. J.; Sabathil, M.; Clark, E. C.; Kress, A.; Schuh, D.; Bichler, M.; Abstreiter, G. and Finley, J. J. Phys. Rev. Lett. 2005, 94, 057402.

(5) Swart, I.; Liljeroth, P. and Vanmaekelbergh, D. Chem. Rev. 2016, 116, 11181-11219.

(6) Ota, T.; Rontani, M.; Tarucha, S.; Nakata, Y.; Song, H. Z.; Miyazawa, T.; Usuki, T.; Takatsu, M. and Yokoyama, N. Phys. Rev. Lett. 2005, 95, 236801.

(7) Ledentsov, N. N.; Shchukin, V. A.; Grundmann, M.; Kirstaedter, N.; Bohrer, J.; Schmidt, O.; Bimberg, D.; Ustinov, V. M.; Egorov, A. Y.; Zhukov, A. E.; Kopev, P. S.; Zaitsev, S. V.; Gordeev, N. Y.; Alferov, Z. I.; Borovkov, A. I.; Kosogov, A. O.; Ruvimov, S. S.; Werner, P.; Gosele, U. and Heydenreich, J. Phys. Rev. B 1996, 54, 8743-8750. 
(8) Scheibner, M.; Yakes, M.; Bracker, A. S.; Ponomarev, I. V.; Doty, M. F.; Hellberg, C. S.; Whitman, L. J.; Reinecke, T. L. and Gammon, D. Nat. Phys. 2008, 4, 291-295.

(9) Scheibner, M.; Bracker, A. S.; Kim, D. and Gammon, D. Solid State Commun. 2009, 149, 1427-1435.

(10) Delley, Y. L.; Kroner, M.; Faelt, S.; Wegscheider, W. and İmamoğlu, A. Phys. Rev. B 2017, $96,241410$.

(11) Bracker, A. S.; Scheibner, M.; Doty, M. F.; Stinaff, E. A.; Ponomarev, I. V.; Kim, J. C.; Whitman, L. J.; Reinecke, T. L. and Gammon, D. Appl. Phys. Lett. 2006, 89, 233110.

(12) Urbieta, A.; Grandidier, B.; Nys, J. P.; Deresmes, D.; Stievenard, D.; Lemaitre, A.; Patriarche, G. and Niquet, Y. M. Phys. Rev. B 2008, 77, 155313.

(13) Maltezopoulos, T.; Bolz, A.; Meyer, C.; Heyn, C.; Hansen, W.; Morgenstern, M. and Wiesendanger, R. Physical Review Letters 2003, 91, 196804.

(14) Teichmann, K.; Wenderoth, M.; Pruser, H.; Pierz, K.; Schumacher, H. W. and Ulbrich, R. G. Nano Lett. 2013, 13, 3571-3575.

(15) Girard, J.-C.; Lemaitre, A.; Miard, A.; David, C. and Wang, Z. Z. J. Vac. Sci. Technol. B 2009, 27, 891-894.

(16) Fain, B.; Robert-Philip, I.; Beveratos, A.; David, C.; Wang, Z. Z.; Sagnes, I. and Girard, J. C. Phys. Rev. Lett. 2012, 108, 126808.

(17) Freitag, N. M.; Reisch, T.; Chizhova, L. A.; Nemes-Incze, P.; Holl, C.; Woods, C. R.; Gorbachev, R. V.; Cao, Y.; Geim, A. K.; Novoselov, K. S.; Burgdörfer, J.; Libisch, F. and Morgenstern, M. Nat. Nanotechnol. 2018, 13, 392-397.

(18) Koenraad, P. M.; Bruls, D. M.; Davies, J. H.; Gill, S. P. A.; Long, F.; Hopkinson, M.; Skolnick, M. and Wolter, J. H. Phys. E 2003, 17, 526-532. 
(19) Legrand, B.; Grandidier, B.; Nys, J. P.; Stievenard, D.; Gerard, J. M. and Thierry-Mieg, V. Appl. Phys. Lett. 1998, 73, 96-98.

(20) Suzuki, K.; Kanisawa, K.; Janer, C.; Perraud, S.; Takashina, K.; Fujisawa, T. and Hirayama, Y. Phys. Rev. Lett. 2007, 98, 136802.

(21) Folsch, S.; Martinez-Blanco, J.; Yang, J. S.; Kanisawa, K. and Erwin, S. C. Nat. Nanotechnol. 2014, 9, 505-508.

(22) Feenstra, R. M.; Stroscio, J. A. and Fein, A. P. Surf. Sci. 1987, 181, 295-306.

(23) Feenstra, R. M. J. Vac. Sci. Technol. B 2003, 21, 2080-2088.

(24) Gong, M.; Duan, K. M.; Li, C. F.; Magri, R.; Narvaez, G. A. and He, L. X. Phys. Rev. B 2008, 77, 045326.

(25) Steiner, D.; Aharoni, A.; Banin, U. and Millo, O. Nano Lett. 2006, 6, 2201-2205.

(26) Liljeroth, P.; Overgaag, K.; Urbieta, A.; Grandidier, B.; Hickey, S. G. and Vanmaekelbergh, D. Phys. Rev. Lett. 2006, 97, 096803.

(27) Schliwa, A.; Winkelnkemper, M. and Bimberg, D. Phys. Rev. B 2007, 76, 205324.

(28) Vasanelli, A.; De Giorgi, M.; Ferreira, R.; Cingolani, R. and Bastard, G. Phys. E 2001, 11, 41-50.

(29) Slot, M. R.; Kempkes, S. N.; Knol, E. J.; van Weerdenburg, W. M. J.; van den Broeke, J. J.; Wegner, D.; Vanmaekelbergh, D.; Khajetoorians, A. A.; Morais Smith, C. and Swart, I. Phys. Rev. X 2019, 9, 011009.

(30) Papatryfonos, K.; Rodary, G.; David, C.; Lelarge, F.; Ramdane, A. and Girard, J. C. Nano Lett. 2015, 15, 4488-4497.

(31) Korkusinski, M. and Hawrylak, P. Phys. Rev. B 2001, 63, 195311.

(32) Szafran, B.; Bednarek, S. and Adamowski, J. Phys. Rev. B 2001, 64, 125301. 
(33) Bester, G.; Shumway, J. and Zunger, A. Phys. Rev. Lett. 2004, 93, 047401.

(34) Fonseca, L. R. C.; Jimenez, J. L. and Leburton, J. P. Phys. Rev. B 1998, 58, 9955-9960. 